\title{
A Multi-Scale Localization Approach to an Ensemble Kalman filter
}

\author{
Takemasa Miyoshi ${ }^{1,2,3}$ and Keiichi Kondo ${ }^{4,1}$ \\ ${ }^{1}$ RIKEN Advanced Institute for Computational Science, Kobe, Japan \\ ${ }^{2}$ University of Maryland, College Park, Maryland, USA \\ ${ }^{3}$ Earth Simulator Center, Japan Agency for Marine-Earth Science and Technology, Yokohama, Japan \\ ${ }^{4}$ University of Tsukuba, Tsukuba, Japan
}

\begin{abstract}
Ensemble data assimilation methods have been improved consistently and have become a viable choice in operational numerical weather prediction. A number of issues for further improvements have been explored, including flow-adaptive covariance localization and advanced covariance inflation methods. Dealing with multi-scale error covariance is among the unresolved issues that would play essential roles in analysis performance. With higher resolution models, generally narrower localization is required to reduce sampling errors in ensemble-based covariance between distant locations. However, such narrow localization limits the use of observations that would have larger-scale information. Previous attempts include successive covariance localization by F. Zhang et al. who proposed applying different localization scales to different subsets of observations. The method aims to use sparse radiosonde observations at a larger scale, while using dense Doppler radar observations at a small scale simultaneously. This study aims to separate scales of the analysis increments, independently of observing systems. Inspired by M. Buehner, we applied two different localization scales to find analysis increments at the two separate scales, and obtained improvements in simulation experiments using an intermediate AGCM known as the SPEEDY model.
\end{abstract}

(Citation: Miyoshi, T., and K. Kondo, 2013: A multi-scale localization approach to an ensemble Kalman filter. SOLA, 9, 170-173, doi:10.2151/sola.2013-038.)

\section{Introduction}

The atmosphere contains many scales by nature, and interactions between different scales play an essential role in atmospheric dynamics. It is generally essential to consider the nature of atmospheric dynamics in data assimilation. Ideally, the ensemble Kalman filter (EnKF) considers multi-scale error covariance explicitly based on the ensemble forecasting. However, due to the limit to the ensemble size, it is widely known that the ensemblebased error covariance is subject to a significant sampling error.

Covariance localization is a widely applied, ad hoc treatment to the sampling error problem (e.g., Houtekamer and Mitchell 1998; Hamill et al. 2001). Assuming that the error covariance becomes smaller in the distance, localization limits the influence of observations within a prescribed radius of influence and reduces sampling errors. Figure 1a illustrates the raw ensemble-based error correlations in an identical twin experiment using an intermediate Atmospheric General Circulation Model (AGCM) known as the SPEEDY model (Molteni 2003) and the Local Ensemble Transform Kalman Filter (LETKF, Hunt et al. 2007). The SPEEDY model has a T30/L7 resolution (horizontal spectral truncation at 30 wave numbers and 7 vertical levels). With a 20 -member ensemble, we can find long-range error correlations from the star point at the center; the long-range error correlations are likely to be sampling noise, as we would not expect such significant long-range analysis

Corresponding author: Takemasa Miyoshi, Data Assimilation Research Team, RIKEN Advanced Institute for Computational Science, 7-1-26 Minatojima-minami-machi, Chuo-ku, Kobe, 650-0047, Japan. E-mail: takemasa.miyoshi@riken.jp. (C2013, the Meteorological Society of Japan. (a)

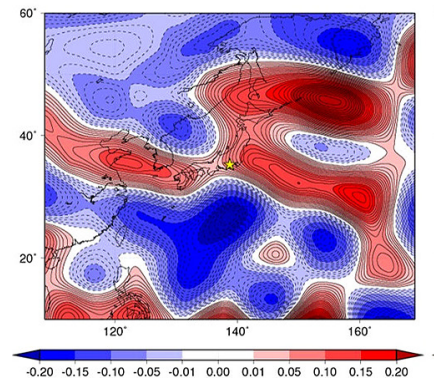

(b)

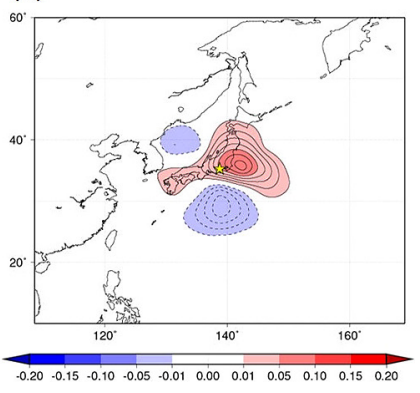

Fig. 1. Zonal wind analysis increments $\left(\mathrm{m} \mathrm{s}^{-1}\right)$ at the 4 th model level $(\sim 500$ $\mathrm{hPa}$ ) from a single profile observation at the center location (yellow star) using the SPEEDY model, illustrating the spatial patterns of ensemblebased error correlations from the center location: (a) raw ensemble-based correlations, and (b) the same as (a) but localized by the Gaussian localization function $($ sigma $=500 \mathrm{~km})$.

increments from the single-point observation. Therefore, a distance-dependent localization function is usually applied, so that we end up with using the localized error correlations as illustrated in Fig. 1b. We can see that the single-point observation at the star point produces analysis increments only in the surrounding area. The analysis increments in Fig. 1b appear to be more realistic intuitively than those in Fig. 1a.

In a high resolution model, high-frequency noise may contaminate the error covariance even in a short range. Previous studies (e.g., Miyoshi and Aranami 2006) suggest that a physically narrower localization function be generally needed for a higher resolution model. The localization scales may be similar in model grid space, so that smaller grid spacing suggests a physically smaller localization scale. This may be problematic when the model grid spacing becomes less than $10 \mathrm{~km}$, and the radius of influence becomes smaller than several tens of kilometers. In such a case, radiosonde observations, for example, will be used only within up to a $\sim 50-\mathrm{km}$ range, too narrow compared with the synoptic scale; synoptic radiosonde observations are spaced typically every $\sim 300 \mathrm{~km}$.

Zhang et al. (2009) proposed so-called successive covariance localization to deal with the narrow localization problem due to a high resolution model. They treated high-resolution Doppler radar observations with a narrow localization scale, while treating synoptic radiosonde observations with a wide localization scale. In this way, a small number of synoptic observations have a large radius of influence although the sampling error is included in the distance, while the sampling error is reduced for a large number of high-resolution Doppler radar observations. The successive localization approach gave promising results.

Covariance localization aims to reduce sampling errors in the ensemble-based error covariance. In most implementations of EnKF, we apply localization to the error covariance between model space and observation space. The observation operator may include spatial or temporal averaging, but otherwise, ideally, localization should be independent of observation types.

Buehner (2012) proposed spatial/spectral covariance localization in which band-pass filters are applied for scale separation. For the longer-range covariance, this is equivalent to applying spatial 
smoothing. If we assume that sampling errors appear randomly in space, spatial smoothing will cancel out the sampling errors, and smaller signals may be extracted. In this study, we follow the spatial/spectral localization and apply spatial smoothing for minimizing the sampling error in the longer-range error covariance. As the first step, two localization scales are considered in this study, and the dual-localization method is implemented and tested with the SPEEDY-model-based LETKF system (Miyoshi 2005). Section 2 describes methodology, followed by numerical experiments in Section 3. Section 4 summarizes the results and provides relevant discussions.

\section{Multi-scale localization approach}

The dual-localization approach is designed to consider two different scales simultaneously. For the smaller scale, we use the raw ensemble perturbations and apply narrower localization. Figure $1 \mathrm{~b}$ illustrates a smaller-scale, short-range error correlations computed from a 20-member ensemble, using the Gaussian localization function $($ sigma $=500 \mathrm{~km})$. Here, we use the ensemble perturbations from an identical twin experiment using the SPEEDY-LETKF system.

For the larger scale, we follow Buehner (2012) and apply spatial smoothing to ensemble perturbations. This spatial smoothing would filter out high-frequency sampling error, and will allow using broader localization without contamination. Figure 2a illustrates the same analysis increments as Fig. 1, but with a $1000-\mathrm{km}$ localization scale. This includes longer-range correlations, with higher-frequency components. If we apply spatial smoothing to filter out higher-frequency components than 21 wave numbers, we obtain Fig. 2b. The SPEEDY model has the horizontal resolution of up to 30 wave numbers. Figure $2 \mathrm{~b}$ has a broader structure in the longer range, although the finer structure near the observed location is lost.

The goal of the proposed approach is to merge the fine shortrange structure (Fig. 1b) and broad long-range structure (Fig. 2b). (a)

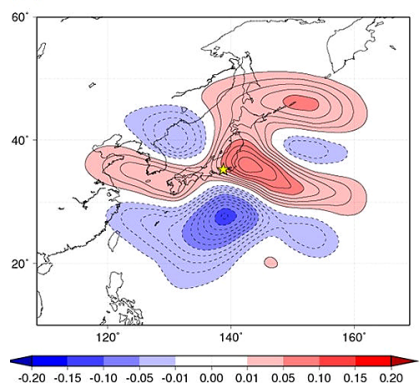

(b)

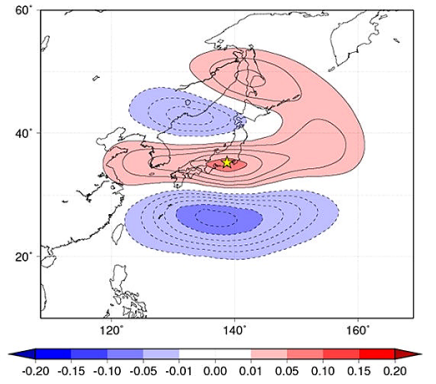

Fig. 2. Similar to Fig. 1, but localized by the Gaussian localization function ( $\operatorname{sigma}=1000 \mathrm{~km}$ ) using (a) the same ensemble perturbations as Fig. 1, and (b) smoothed ensemble perturbations. (a)

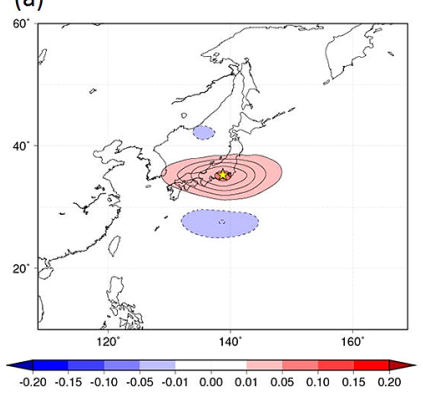

(b)

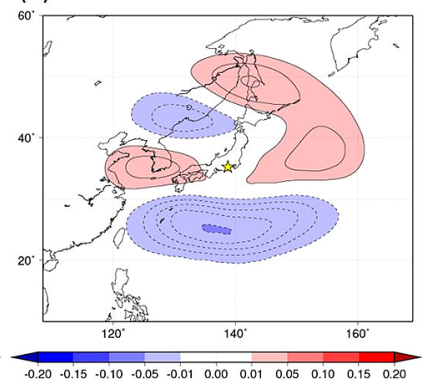

Fig. 3. Similar to Fig. 2b, but (a) using the 500-km Gaussian localization function, and (b) the difference between Figs. $2 \mathrm{~b}$ and $3 \mathrm{a}$.

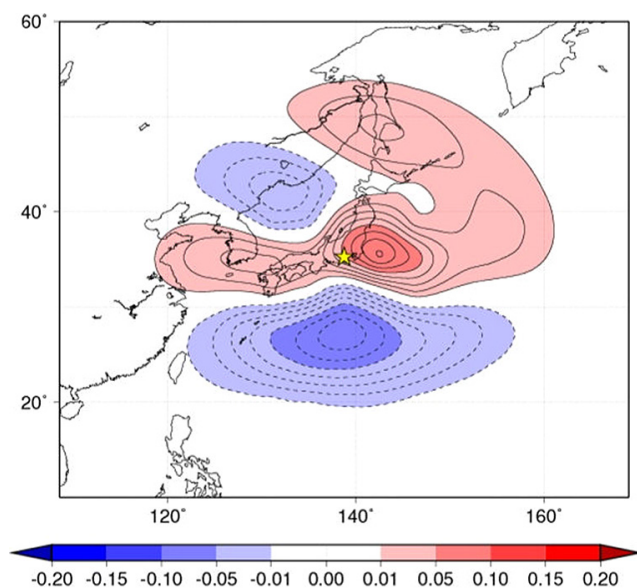

Fig. 4. Similar to Figs. 1-3, but multi-scale error correlations.

A simple approach may be taking a weighted mean of Fig. 1b and Fig. $2 b$, but that will reduce the long-range signals. In this study, we take an alternative approach, so that we use Fig. 1b for the short range, and Fig. $2 b$ only for the long range. That is, we take out the shorter-range component of smoothed correlations from Fig. $2 \mathrm{~b}$. This is obtained by applying the $500-\mathrm{km}$ localization to the correlations based on the smoothed ensemble perturbations (Fig. 3a). If we subtract Fig. 3a from Fig. 2b, we obtain Fig. 3b, which gives the longer-range correlations. To this end, we may simply add the short-range component Fig. $1 \mathrm{~b}$ and long-range component Fig. 3b, and obtain the merged multi-scale correlations Fig. 4. This contains the fine structure near the observed location, while having the longer-range component without high-frequency noise.

In summary, the algorithm of the proposed dual-localization approach is as follows:

1. Compute the analysis increments regularly with smaller-scale localization (Fig. 1b)

2. Compute the analysis increments with larger-scale localization using smoothed ensemble perturbations (Fig. 2b)

3. Compute the analysis increments with smaller-scale localization using smoothed ensemble perturbations (Fig. 3a)

4. Take the difference between 2 and 3 (Fig. 3b)

5. Add 1 and 4 (Fig. 4)

\section{Experiments}

Identical twin experiments, also known as observing system simulation experiments (OSSEs) under the perfect model scenario, are performed with the SPEEDY-LETKF system (Miyoshi 2005). The nature run is generated by running the SPEEDY model from the standard atmosphere at rest, and the first year is considered as a spin up. The first day of the nature run is arbitrarily assigned at 0000 UTC 1 January 1981. Observation data are simulated by adding independent Gaussian random errors to the nature run every 6 hours at radiosonde-like stations (Miyoshi 2005). Observations are denser over the populated areas such as the East Asia, Europe, and North America, and sparser over the ocean. Simulated observations include wind components (U, V) and temperature $(\mathrm{T})$ at all 7 levels, specific humidity $(\mathrm{Q})$ from the bottom to the 4th level, and surface pressure (Ps) only at the surface. The observation error standard deviations are fixed at $1.0 \mathrm{~m} \mathrm{~s}^{-1}$ for $\mathrm{U}$ and $\mathrm{V}, 1.0 \mathrm{~K}$ for T, $1.0 \mathrm{~g} \mathrm{~kg}^{-1}$ for Q, and $1.0 \mathrm{hPa}$ for Ps. 20 ensemble members are employed, and the initial ensemble conditions are chosen from the nature run in January 1983, so that the initial ensemble members have no meaningful correlation to the nature run on 1 January 1981. The adaptive covariance inflation method of Miyoshi (2011) is applied to avoid manual optimization of the inflation parameter. 
(a)

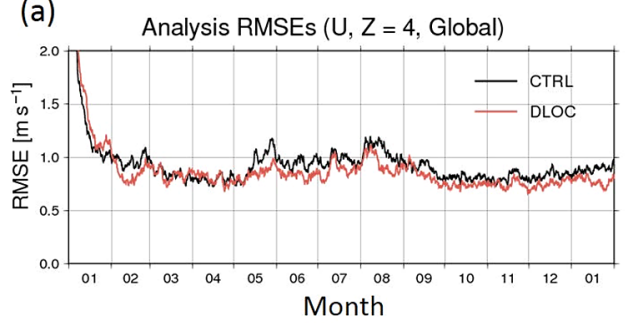

(c)

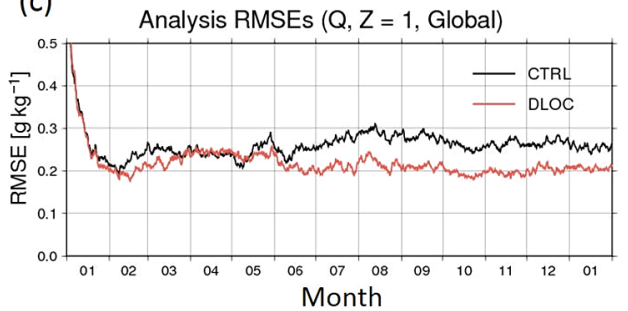

(b)

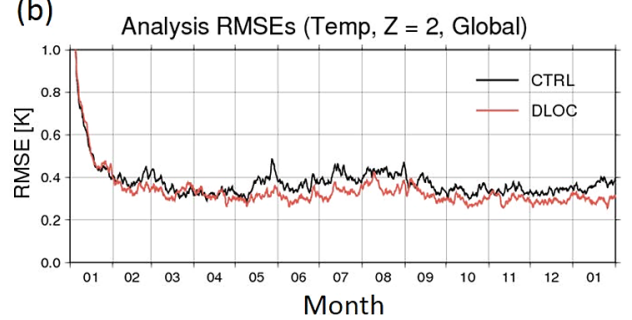

(d)

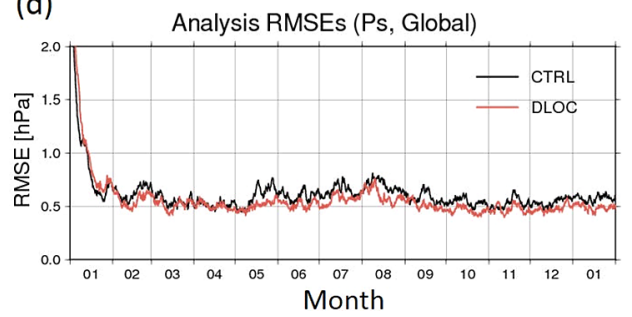

Fig. 5. Time series of analysis RMSE for (a) zonal wind $\left(\mathrm{m} \mathrm{s}^{-1}\right)$ at the 4 th model level $(\sim 500 \mathrm{hPa})$, (b) temperature (K) at the $2 \mathrm{nd}$ model level $(\sim 850 \mathrm{hPa})$, (c) specific humidity $\left(\mathrm{g} \mathrm{kg}^{-1}\right)$ at the lowest level $(\sim 950 \mathrm{hPa})$, and (d) surface pressure (hPa). Black and red curves indicate CTRL and DLOC experiments, respectively. Abscissa indicates the month.

(a)

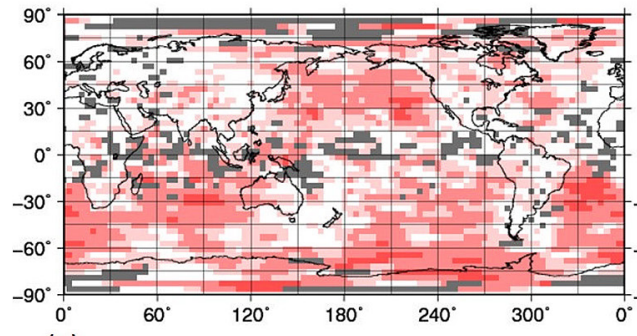

(c)

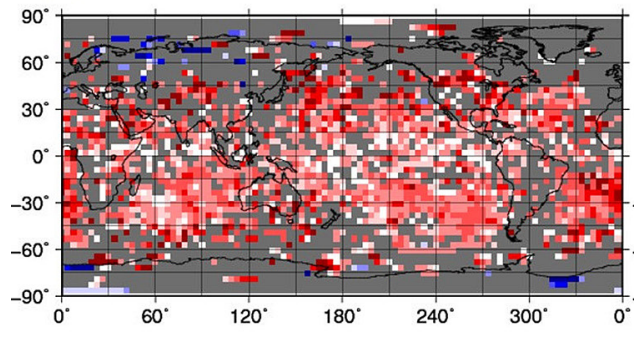

(b)

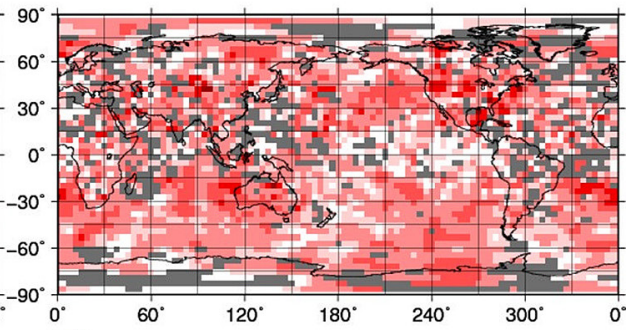

(d)

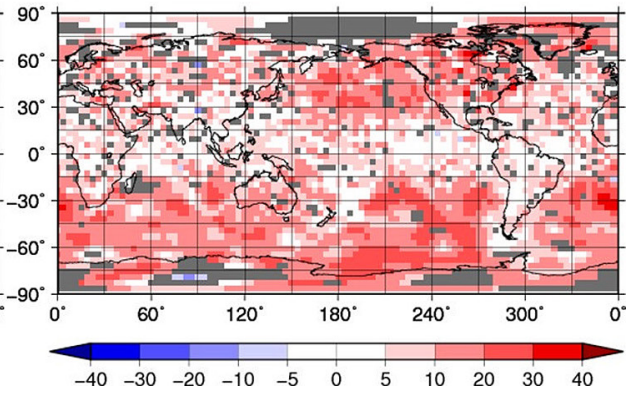

Fig. 6. Improvements (\%) of 1-year-average analysis RMSE of DLOC over CTRL for (a) zonal wind at the 4th model level ( $\sim 500 \mathrm{hPa})$, (b) temperature (K) at the 2nd model level ( $\sim 850 \mathrm{hPa})$, (c) specific humidity at the lowest level ( $\sim 950 \mathrm{hPa})$, and (d) surface pressure. Red (blue) indicates advantage (disadvantage) of DLOC.

Two experiments are performed to investigate the impact of the proposed dual localization approach. The control experiment (CTRL) uses single 700-km Gaussian localization after manual tuning. This localization scale is the same as what Kang et al. (2011, 2012) used to study carbon cycle data assimilation using the SPEEDY-LETKF system. Other choices of the localization scale parameter such as $500 \mathrm{~km}$ and $900 \mathrm{~km}$ did not give better results than CTRL. The other experiment (DLOC) employs the dual-localization method with $500-\mathrm{km}$ and $900-\mathrm{km}$ localization scales. These two scales are chosen after manual tuning. For spatial smoothing of ensemble perturbations, the spherical harmonics spectral truncation at 21 wave numbers is applied. Experiments are performed for 13 months from 1 January 1981 to 1 February 1982, and verifications are performed for the latter 12 months (1 February 1981 to 1 February 1982) assuming 1-month spin-up period. For statistical significance, four parallel experiments are performed using different realizations of observational noise but with the same observation error standard deviations. Namely, the four experiments are basically identical except the random numbers for observation noise.

First, the root mean square errors (RMSE) are investigated. The RMSE are defined as the RMS difference from the nature run for each variable at each vertical level. Figure 5 shows time series of analysis RMSE for an experiment, although the other three parallel experiments show very similar results. We find overall advantage of dual localization (DLOC) over the manually-tuned single localization (CTRL). After about 4-month run (May 1981 and later), DLOC shows very clear advantage, particularly for humidity. If we look at the spatial pattern, we find advantage of DLOC almost everywhere (Fig. 6). Figure 6 greys out nonsignificant areas in which any one of the four parallel experiments disagrees. We find general advantage of DLOC over the ocean in 
(a)

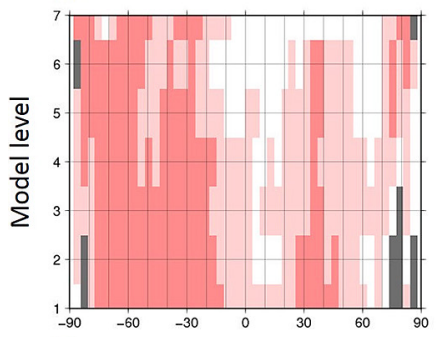

(c)

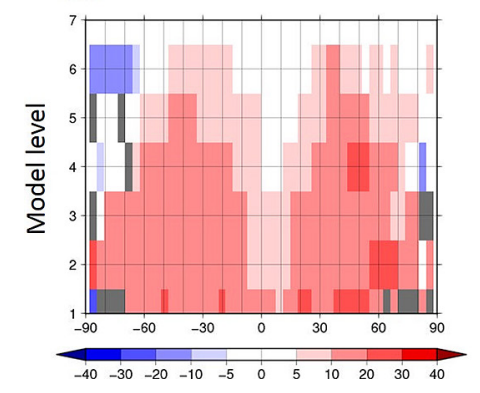

Fig. 7. Similar to Fig. 6, but for zonal-average vertical structure of (a) zonal wind, (b) temperature, and (c) specific humidity.

the mid-latitudes, probably because of the inclusion of the broader localization over the sparsely-observed area. In fact, $900-\mathrm{km}$ single localization gives more accurate analysis over the ocean than CTRL, but still worse than DLOC. Since 900-km localization gives less accurate analysis over land than CTRL, the overall analysis accuracy of $900-\mathrm{km}$ localization is not better than CTRL. The advantage of DLOC is generally true to other vertical levels (Fig. 7).

\section{Summary and discussion}

Multi-scale error covariance localization would be essential in EnKF with a higher-resolution model, and the dual-localization method was proposed in this study, inspired by Buehner (2012). The identical twin experiments or the perfect-model OSSEs using the SPEEDY-LETKF system showed significant advantage of the dual-localization method over the traditional single localization method.

The dual-localization method requires solving the LETKF analysis equations three times. This may slow down the computations of the LETKF analysis updates by a factor of up to three. However, the computational cost for the ensemble prediction part, which is usually the major part, remains the same. Also, we can achieve better analysis accuracy by the additional computations. Namely, we have a choice of spending more CPU time for the additional accuracy. It may or may not be worth spending the additional computer resources, depending on the application. Probably for numerical weather prediction at a higher resolution, it may be essential to consider multi-scale localization at additional cost.

The dual-localization method in this study considered two localization scales. However, it is straightforward to extend the method to three or more scales. Following the dual-localization approach, considering $n$ localization scales requires solving the LETKF analysis updates by $(2 n-1)$ times. Each additional scale requires two additional LETKF updates in order to make the donut-shape analysis increments for the additional scale, just in the same way as the dual-localization approach. Testing more than two scales makes more sense with more realistic models with more multi-scale structures, and is a subject of future research.

Another limitation of this study is that only horizontal localization was considered. Multi-scale phenomena may also have multi-scale structures in the vertical and temporal error covariance. We may apply a similar multi-scale localization approach to vertical localization by applying spatial smoothing in the vertical, but temporal smoothing may not be as simple. Further considerations of vertical and temporal multi-scale localization require more realistic high-resolution applications, and remains to be a subject of future study.

\section{Acknowledgements}

The authors thank the members of the University of Maryland Weather-Chaos Group and RIKEN Data Assimilation Research Team for fruitful discussions. This study was partially supported by the Office of Naval Research (ONR) grant N000141010149 under the National Oceanographic Partnership Program (NOPP), and by the Japan Society for the Promotion of Science (JSPS) KAKENHI grant 241335.

\section{References}

Buehner, M., 2012: Evaluation of a spatial/spectral covariance localization approach for atmospheric data assimilation. Mon. Wea. Rev., 140, 617-636.

Hamill, T. M., J. S. Whitaker, and C. Snyder, 2001: Distancedependent filtering of background error covariance estimates in an ensemble Kalman filter. Mon. Wea. Rev., 129, 27762790 .

Houtekamer, P. L., and H. L. Mitchell, 1998: Data assimilation using an ensemble Kalman filter technique. Mon. Wea. Rev., 126, 796-811.

Hunt, B. R., E. J. Kostelich, and I. Szunyogh, 2007: Efficient data assimilation for spatiotemporal chaos: a local ensemble transform Kalman filter. Physica D, 230, 112-126.

Kang, J.-S., E. Kalnay, J. Liu, I. Fung, T. Miyoshi, and K. Ide, 2011: "Variable localization" in an Ensemble Kalman Filter: application to the carbon cycle data assimilation. J. Geophys. Res., 116, D09110, doi:10.1029/2010JD014673.

Kang, J.-S., E. Kalnay, T. Miyoshi, J. Liu, and I. Fung, 2012: Estimation of surface carbon fluxes with an advanced data assimilation methodology. J. Geophys. Res., 117, D24101. doi:10.1029/2012JD018259.

Miyoshi, T., 2005: Ensemble Kalman filter experiments with a primitive-equation global model, Ph.D. dissertation, University of Maryland, College Park, $197 \mathrm{pp}$.

Miyoshi, T., 2011: The gaussian approach to adaptive covariance inflation and its implementation with the local ensemble transform Kalman filter. Mon. Wea. Rev., 139, 1519-1535, doi:10.1175/2010MWR3570.1.

Miyoshi, T., and K. Aranami, 2006: Applying a four-dimensional local ensemble transform Kalman filter (4D-LETKF) to the JMA nonhydrostatic model (NHM). SOLA, 2, 128-131, doi:10.2151/sola.2006-033.

Molteni, F., 2003: Atmospheric simulations using a GCM with simplified physical parametrizations. I: model climatology and variability in multi-decadal experiments. Clim. Dyn., 20, 175-191.

Zhang, F., Y. Weng, J. A. Sippel, Z. Meng, and C. H. Bishop, 2009: Cloud-resolving hurricane initialization and prediction through assimilation of Doppler radar observations with an ensemble Kalman filter. Mon. Wea. Rev., 137, 2105-2125.

Manuscript received 29 August 2013, accepted 8 October 2013 SOLA: http://www.jstage.jst.go.jp/browse/solal 